\title{
NICE SETS OF MULTI-INDICES
}

\author{
W. R. MADYCH AND P. SZEPTYCKI ${ }^{1}$
}

\begin{abstract}
Finite sets, $A$, of $n$-tuples for which $\left(\Sigma_{\alpha \in A}\left(\mathbb{I}_{j=1}^{n}\left|x_{j}\right|^{\alpha}\right)\right)^{-p}, p>0$, is integrable over $R^{n}$ are given a simple characterization. Applications to certain Fourier multiplier theorems are mentioned.
\end{abstract}

Let $A$ be a finite subset of $R^{n}$ and consider the function $h_{A}$, defined on $R^{n}$ by the formula $h_{A}(x)=\sum_{\alpha \in A}\left(\prod_{i=1}^{n}\left|x_{i}\right|^{\alpha_{i}}\right)$. It is sometimes useful to know when $h_{A}^{-p}, p>0$, is integrable over $R^{n}$. When this is the case we call $A$ $p$-nice.

For example consider those finite subsets $A$ of $R^{n}$ whose elements, $\alpha$, have components which are nonnegative integers and associate with each such $\alpha$ the derivative of order $\alpha$ in the usual manner. Given a distribution $f$ on $R^{n}$, the integrability of $h_{A}^{-2}$ determines the derivatives of $f$ in $L^{2}\left(R^{n}\right)$ needed to conclude that $f$ is a continuous function as in a classical theorem of Sobolev. The integrability of $h_{A}^{-2}$ also determines the derivatives one needs to compute in order to apply certain variants of the Fourier multiplier theorem of Marcinkiewicz (see [2]).

Let $v$ denote that element of $R^{n}$ all of whose components are one.

Proposition. $A$ finite subset $A$ of $R^{n}$ is $p$-nice if and only if $v / p$ is contained in the interior of the convex hull of $A$.

To see that the condition on $v / p$ is sufficient let $e_{j}, j=1, \ldots, 2^{n}$, denote those elements of $R^{n}$ whose components are either one or minus one. By hypothesis there is a positive $\varepsilon$ such that $(v / p)+\varepsilon e_{j}=\sum_{\alpha \in A} r_{j, \alpha} \alpha$, where $r_{j, \alpha} \geqslant 0$ and $\Sigma_{\alpha \in A} r_{j, \alpha}=1, j=1, \ldots, 2^{n}$. Since $h_{A}(x) \geqslant \sum_{\alpha \in A} r_{j, \alpha}\left(I I\left|x_{j}\right|^{\alpha_{j}}\right)$, an application of the inequality between the arithmetic and geometric mean results in the $2^{n}$ inequalities

$$
\left(h_{A}(x)\right)^{-p} \leqslant \prod_{j=1}^{n}\left(\left|x_{j}\right|^{-1 \pm p \varepsilon}\right) .
$$

It is clear from the above inequalities that $\left(h_{A}(x)\right)^{-p}$ is integrable over $R^{n}$.

Suppose $v / p$ is not contained in the interior of the convex hull of $A$. Then there is a hyperplane which separates $v / p$ and the convex hull of $A$. More specifically, there is a $u$ in $R^{n}$ such that $\alpha \cdot u \leqslant(v \cdot u) / p$ for all $\alpha \in A$.

There is no loss of generality if we assume, which we do, that $u$ and all the

Received by the editors April 14, 1977.

AMS (MOS) subject classifications (1970). Primary 42A16.

'Supported by NSF Grant No. GP1692. 
elements of $A$ have nonnegative components. For if $T$ is the transformation which maps $x=\left(x_{1}, \ldots, x_{n}\right)$ into $\left(\gamma_{1} x_{1}, \ldots, \gamma_{n} x_{n}\right), \gamma_{i} \neq 0, i=1, \ldots, n$, and $B=\{T(\alpha-v / p)+v / p: \alpha \in A\}$, then $\beta \cdot T^{-1} u<\left(v \cdot T^{-1} u\right) / p$ for all $\beta$ in $B$ and

$$
\int_{R^{n}}\left(h_{B}(x)\right)^{-p} d x=\left|\prod_{i=1}^{n} \gamma_{i}\right|^{-1} \int_{R^{n}}\left(h_{A}(x)\right)^{-p} d x .
$$

Furthermore, it is clear that if $u$ and $A$ do not satisfy the desired property then $\gamma_{1}, \ldots, \gamma_{n}$ can be chosen so that $T^{-1} u$ and $B$ do.

If none of the components of $u$ are zero, then $A$ is contained in the convex hull of the origin and the points $\beta_{1}, \ldots, \beta_{n}$, where the $j$ th component of $\beta_{j}$ is $b_{j}=u \cdot v / p u_{j}$ and the remaining components are zero, $j=1, \ldots, n$. Again an application of the inequality between the arithmetic and geometric mean results in $\prod_{j=1}^{n}\left|x_{j}\right|^{\alpha_{j}}<1+\sum_{j=1}^{n}\left|x_{j}\right|^{b_{j}}$, for all $\alpha=\left(\alpha_{1}, \ldots, \alpha_{n}\right)$ in $A$. The last inequality, together with a polar change of variables or direct calculation, implies that

$$
\int_{R^{n}}\left(h_{A}(x)\right)^{-p} d x \geqslant C \int_{0}^{\infty}(1+r)^{p} r^{\lambda-1} d r
$$

where $\lambda=\sum_{j=1}^{n} b_{j}^{-1}$ and $C$ is a positive constant which depends on $A$ and $p$. Since $\sum_{j=1}^{n} b_{j}^{-1}=p$, the last integral diverges.

If one of the components of $u$, say $u_{1}$, is zero, proceed by induction. Namely, given $x$ in $R^{n}$ denote by $x^{\prime}$ that element of $R^{n-1}$ obtained by deleting the first component of $x$ and let $A^{\prime}=\left\{\alpha^{\prime}: \alpha \in A\right\}$. Observe that $\int R^{n}\left(h_{A}(x)\right)^{-p} d x \geqslant \int_{R^{n-1}}\left(h_{A^{\prime}}\left(x^{\prime}\right)\right)^{-p} d x^{\prime}$. Since $v^{\prime} / p$ is not in the interior of the convex hull of $A^{\prime}$, the last integral diverges. This completes the proof.

Some examples of nice sets are given in [5]. An application of the proposition shows that the set $A=\left\{\alpha=\left(\alpha_{1}, \ldots, \alpha_{n}\right): \alpha_{i}=0\right.$ or 1 and $\Sigma \alpha_{i} \leqslant \kappa$, where $\kappa$ is the least integer greater than $n / p\}$ is $p$-nice. (Note that the usage of the term nice in [5], where only sets of multi-indices are considered, is somewhat different from ours. In fact, if a set of multi-indices is $p$-nice and $\alpha \in A$ implies that any multi-index $\beta$ satisfying $\beta \leqslant \alpha$ is also in $A$, we call $A$ very $p$-nice.)

As another application, consider the multiplier theorem of Hormander [2, p. 120] and let $A$ denote the set of multi-indices in the hypothesis of that theorem. Recalling the proof, besides the fact that $A$ is very 2-nice, the only other property of $A$ which is used is that $\int_{|x|>t}\left[h_{A}(x)\right]^{-2} d x \leqslant C t^{-\delta}$, for some positive constants $C$ and $\delta$ and all positive $t$. A similar inequality holds for any $p$-nice set. In fact, more generally, if $S$ is a linear transformation on $R^{n}$ satisfying $S x \cdot x \geqslant|x|^{2}$ and $T_{t}=\exp (S \log t), t>0$, is the group of linear transformations with infinitesimal generator $S$, then we have the following

Corollary. If $A$ is a p-nice set then there are positive constants $C$ and $\delta$ such that $\int_{B_{t}}\left(h_{A}(x)\right)^{-p} d x \leqslant C t^{-\delta}, t>0$, where $B_{t}=\left\{x \in R^{n}:\left|T_{t^{-1}} x\right| \geqslant 1\right\}$.

If $Q_{t}$ denotes the complement of the cube $\left\{x:\left|x_{i}\right| \leqslant t / \sqrt{ } n\right\}$ then $Q_{t} \supset B_{t}$, 
$t>1$, and hence it suffices to verify the inequality with $B_{t}$ replaced by $Q_{t}$. The $2^{n}$ inequalities labeled (1) in the proof of the proposition imply that $h_{A}(x)^{-p}<C_{1} \Pi_{j=1}^{n} h\left(x_{j}\right)$, where $h(s)=(1+|s|)^{-2 \delta}|s|^{-1+\delta}$, for $-\infty<s<$ $\infty$. A direct calculation shows that

$$
\int_{Q_{1}}\left(h_{A}(x)\right)^{-p} d x \leqslant C_{1}\left[2^{n}-1\right]\left(\int_{-\infty}^{\infty} h(s) d s\right)^{n-1} \int_{t / \vee n}^{\infty} s^{-1-\delta} d s=C t^{-\delta} .
$$

It follows from the corollary that Hormander's multiplier theorem and its variants are true when the conditions on the set of multi-indices is somewhat relaxed. (See [1], [3], [4], [6], [7].) As a specific example we mention the following

THEOREM. Suppose $m \in L^{\infty}\left(R^{n}\right), A$ is a very q-nice set of multi-indices, and $\Sigma_{\alpha \in A} \int_{1<|\xi|<2}\left|D^{\alpha} m_{t}(\xi)\right|^{q} d \xi \leqslant B^{q}$ for all $t>0$, where $m_{t}(\xi)=m\left(T_{t} \xi\right)$ and $q$ is a number such that $1 \leqslant q \leqslant 2$. Then $m$ is a Fourier multiplier from $L^{p}\left(R^{n}\right)$ to $L^{p}\left(R^{n}\right)$ with multiplier norm bounded by $C B p^{2}(p-1)^{-1}$ for all $p, 1<p<\infty$, where $C$ is a constant depending only on $n$. Furthermore, if $H^{1}$ is the parabolic Hardy space defined with respect to the group of "dilations" $T_{t}^{*}=$ $\exp \left(S^{*} \log t\right)$, where $S^{*}$ is the transpose of $S$, as in [1], then $m$ is a Fourier multiplier from $H^{1}$ to $H^{1}$ with multiplier norm bounded by $C B$, where $C$ depends only on the choice of norm in $H^{1}$.

\section{REFERENCES}

1. A. P. Calderon and A. Torchinsky, Parabolic maximal functions associated with a distribution. I, Advances in Math. 16 (1975), 1-64; II. (to appear).

2. L. Hormander, Estimates for translation invariant operators in $L^{p}$ spaces, Acta Math. 104 (1960), 93-140.

3. V. M. Kagan, On the boundedness of pseudo-differential operators in $L^{p}$, Izv. Vysš. Učebn. Zaved. Matematika 73 (1968), 35-44. (Russian)

4. W. Littman, Multipliers in $L^{p}$ and interpolation, Bull. Amer. Math. Soc. 71 (1965), 764-766.

5. W. R. Madych, Iterated Littlewood-Paley functions and a multiplier theorem, Proc. Amer. Math. Soc. 45 (1974), 325-331.

6. N. M. Riviere, Singular integrals and multiplier operators, Ark. Mat. 9 (1971), 243-278.

7. P. Szeptycki, A remark on Miklin's theorem about multipliers of Fourier transforms, Notices Amer. Math. Soc. 11 (1964), 558.

Department of Mathematics, Iowa State University, Ames, Iowa 50011

Department of Mathematics, Untversity of Kansas, LaWrence, Kansas 66045 